University of Wollongong

Research Online

Australian Institute for Innovative Materials -

Papers

Australian Institute for Innovative Materials

2013

Levetiracetam-loaded biodegradable polymer implants in the tetanus toxin model of temporal lobe epilepsy in rats

Amy J. Halliday

University of Wollongong

Toni E. Campbell

University of Wollongong, tonicamp@uow.edu.au

Timothy S. Nelson

The Bionic Ear Institute

Karen J. McLean

St Vincent's Hospital Melbourne

Gordon G. Wallace

University of Wollongong, gwallace@uow.edu.au

See next page for additional authors

Follow this and additional works at: https://ro.uow.edu.au/aiimpapers

Part of the Engineering Commons, and the Physical Sciences and Mathematics Commons

Research Online is the open access institutional repository for the University of Wollongong. For further information contact the UOW Library: research-pubs@uow.edu.au 


\title{
Levetiracetam-loaded biodegradable polymer implants in the tetanus toxin model of temporal lobe epilepsy in rats
}

\begin{abstract}
Approximately one-third of people with epilepsy receive insufficient benefit from currently available anticonvulsant medication, and some evidence suggests that this may be due to a lack of effective penetration into brain parenchyma. The current study investigated the ability of biodegradable polymer implants loaded with levetiracetam to ameliorate seizures following implantation above the motor cortex in the tetanus toxin model of temporal lobe epilepsy in rats. The implants led to significantly shorter seizures and a trend towards fewer seizures for up to 1 week. The results of this study indicate that drugeluting polymer implants represent a promising evolving treatment option for intractable epilepsy. Future research is warranted to investigate issues of device longevity and implantation site.
\end{abstract}

\section{Keywords}

tetanus, levetiracetam, toxin, model, temporal, lobe, epilepsy, rats, loaded, polymer, implants, biodegradable

Disciplines

Engineering | Physical Sciences and Mathematics

\section{Publication Details}

Halliday, A. J., Campbell, T. E., Nelson, T. S., McLean, K. J., Wallace, G. G. \& Cook, M. J. (2013).

Levetiracetam-loaded biodegradable polymer implants in the tetanus toxin model of temporal lobe epilepsy in rats. Journal of Clinical Neuroscience, 20 (1), 148-152.

\section{Authors}

Amy J. Halliday, Toni E. Campbell, Timothy S. Nelson, Karen J. McLean, Gordon G. Wallace, and Mark J. Cook 


\section{Title page}

Title:

Levetiracetam-loaded biodegradable polymer cortical implants in the tetanus toxin model of temporal lobe epilepsy.

\section{Authors:}

Amy J. Halliday ${ }^{1,3}$

Toni E. Campbell ${ }^{3}$

Timothy S. Nelson ${ }^{2}$

Karen J. McLean ${ }^{1}$

Gordon G. Wallace ${ }^{3}$

Mark J. Cook ${ }^{1,2}$

\section{Affiliations:}

${ }^{1}$ Centre for Clinical Neurosciences and Neurological Research, St Vincent's Hospital Melbourne, PO Box 2900, Fitzroy, Victoria, Australia 3065

${ }^{2}$ The Bionic Ear Institute, 384-388 Albert St, East Melbourne, Victoria, Australia 3002

${ }^{3}$ Intelligent Polymer Research Institute and ARC Centre of Excellence for Electromaterials Science, AllM Facility, Innovation Campus, University of Wollongong, Wollongong, New South Wales, Australia 2522

\section{Corresponding author:}

Amy Halliday

Centre for Clinical Neurosciences and Neurological Research

$5^{\text {th }}$ Floor, Daly Wing

St Vincent's Hospital

35 Victoria Pde

Fitzroy VIC 3065

Australia

Telephone: +61 429885776

Fax: +61392883350

email: amyjhalliday@gmail.com

\section{Sources of support}

- the Victorian Government's Science Technology and Innovation Initiative administered by the Department of Industry, Innovation and Regional Development

- the Victorian Government through its Operational Infrastructure Support Program and the Helen Macpherson Smith Trust, to the Bionic Ear Institute 


\section{Abstract}

Approximately one third of people with epilepsy receive insufficient benefit from currently available anticonvulsant medication, and some evidence suggests that this may be due to a lack of effective penetration into brain parenchyma. The current study investigated the ability of biodegradable polymer implants loaded with Levetiracetam to ameliorate seizures following implantation above the motor cortex in the tetanus toxin model of temporal lobe epilepsy in rats. The implants led to significantly shorter seizures and a trend towards fewer seizures for up to one week. The results of this study indicate that drug-eluting polymer implants represent a promising evolving treatment option for intractable epilepsy. Future research is warranted to investigate issues of device longevity and implantation site.

\section{Key words}

Polymer, levetiracetam, epilepsy, anticonvulsant, tetanus toxin, biodegradable, drug delivery, implantable devices. 


\section{Introduction}

Epilepsy is among the most common chronic neurological disorders, affecting nearly $1 \%$ of the population. Epilepsy is well recognised for being highly refractory to medical treatment, with up to one third of patients receiving no benefit from anticonvulsant medication.

A range of treatment options are currently being investigated aimed at improving the availability of anticonvulsant drugs to the brain. One such treatment option is the intracranial implantation of polymer-based drug delivery systems. These implants are made of materials that slowly break down following implantation, gradually releasing their drug load into the implant site. Recent studies have demonstrated some success at using similar biodegradable implants to treat animal models of several neurological disorders with focal pathologies such as Parkinson's disease, Huntington's disease and Alzheimer's disease[1]; however, their application to animal models of epilepsy have to date been less successful.

The most intensely investigated biodegradable polymer in intracranial drug delivery for neurological disorders has been the copolymer poly-lactide-co-glycolide (PLGA). PLGAbased implants have been shown to be very well tolerated by the brain in animal models of neurological disorders ${ }^{[2-7]}$. Given their biocompatibility and success in animal models of other focal neurological disorders, we sought to investigate the efficacy of PLGA sheets loaded with the novel anticonvulsant drug Levetiracetam (LEV) in an animal model of epilepsy. In this investigation, sheets of PLGA loaded with LEV were developed and their ability to ameliorate seizures was investigated in the hippocampal tetanus toxin model of mesial temporal lobe epilepsy (TLE) in rats.

\section{Methods}




\subsection{Animals}

Adult male Sprague-Dawley rats were obtained from FlindersUniversity (Adelaide, SA, Australia) and housed individually in $12 \mathrm{~h}$ light/dark cycles with ad libitum access to food and water. All experiments were approved by St Vincent's Hospital (Melbourne) Animal Ethics Committee and conducted in accordance with the Australian Code of Practice for the Care and Use of Animals for Scientific Purposes (2004).

\subsection{Polymer production}

Polymer sheets were produced by pipetting $1 \mathrm{~mL}$ of a solution of PLGA 85:15 (2\% w/v; SigmaAldrich, Australia) and LEV (4\% w/v; gift from UCB Pharma, Belgium) in dichloromethane (SigmaAldrich, Australia) into a Teflon mold. The solvent was evaporated overnight, resulting in a $2 \mathrm{~cm}^{2}$ film with a theoretical LEV loading of $67 \% \mathrm{w} / \mathrm{w}$. The sheets were cut to size $(4 \mathrm{~mm} \times 2 \mathrm{~mm})$ immediately before implantation.

\subsection{In vitro drug release}

Discs of $2.5 \mathrm{~mm}$ diameter were cut from the LEV films, placed in vials of $350 \mu \mathrm{L}$ of artificial cerebrospinal fluid (aCSF) and incubated in a water bath at 37C. At set time points, three vials were removed from the water bath and the concentration of LEV in the sample determined using high performance liquid chromatography (HPLC).

Artificial cerebral spinal fluid contained sodium chloride ( $\mathrm{NaCl} ; 0.866 \% \mathrm{w} / \mathrm{v})$, potassium chloride $(\mathrm{KCl} ; 0.224 \% \mathrm{w} / \mathrm{v})$, calcium dichloride $(\mathrm{CaCl} 2.2 \mathrm{H} 2 \mathrm{O} ; 0.0206 \% \mathrm{w} / \mathrm{v})$ and magnesium dichloride (MgCl2.6H2O; 0.0164\% w/v) in $1 \mathrm{mM}$ phosphate buffer (pH 7.4).

\subsection{Implantation surgery}

Two days prior to surgery, rats were administered oral antibiotics to assist in the prevention of infection following surgery (Baytril, $3.5 \mathrm{~mL} / \mathrm{L}$ in drinking water). Immediately prior to surgery, rats were anaesthetized with an i.p. injection of ketamine $(70 \mathrm{mg} / \mathrm{kg})$ and xylazine (10 mg/kg), and administered Carprofen (5 mg/kg, s.c.) for pain relief. Once anaesthetized, 
rats were placed in a stereotaxic apparatus, and administered isoflurane $(0.5 \%$ in oxygen, 2 $\mathrm{L} / \mathrm{min}$ ) via a nose-cone. $50 \mathrm{ng}$ of tetanus toxin in $0.5 \mu \mathrm{L}$ of phosphate-buffered saline was injected into the right hippocampus (AP: $-3.5 \mathrm{~mm}$; ML: + $3.0 \mathrm{~mm}$; DV: $-3.5 \mathrm{~mm}$ from Bregma) via a microsyringe at a rate of $1 \mu \mathrm{L} / \mathrm{min}$. The syringe was left in place for 5 min before being slowly retracted. A craniotomy was created over the left motor and somatosensory cortices ( $5 \mathrm{~mm} \times 3 \mathrm{~mm}$, centred over AP: $0 \mathrm{~mm}, \mathrm{ML}:-4.0 \mathrm{~mm}$ from Bregma) and a 16 channel microwire array electrode was implanted within the motor cortex (Tucker-Davis Technologies (TDT), USA). A second craniotomy was created above the right motor cortex (5 $\mathrm{mm} \times 5 \mathrm{~mm}$, centered over AP: $0 \mathrm{~mm}, \mathrm{ML}:+4.0 \mathrm{~mm}$ from Bregma), the central section of bone removed and a flap cut in the exposed dura. A sheet of polymer was placed on the exposed brain, and the dural flap and bone piece replaced. Control rats received a craniotomy without implantation of a polymer $(n=10)$ while experimental rats received polymers containing LEV $(n=11)$. The polymer craniotomy site was sealed with an alginate-based hydrogel and the entire surgical site covered with dental cement and sutured closed. The animal was placed on a heat pad for recovery.

\subsection{EEG monitoring}

Beginning three days after surgery, rats were monitored for at least two hours on at least three days per week for three weeks following surgery. Rats were anaesthetised with isoflurane ( $4 \%$ in oxygen, $2 \mathrm{~L} / \mathrm{min}$ ), and a shielded cable was used to connect the electrode to the electroencephalogram (EEG) acquisition system, which consisted of TDT processors and high impedance head stages driven by custom-designed software. The rat was allowed to recover fully from the anesthetic before recording began so that EEGs were obtained from freely moving rats.

\subsection{Data analysis}

ECoGs were visualized using a custom-designed MATLAB programme, and used to determine the duration of each seizure (seconds) and seizure frequency (seizures/hour). 
Seizure severity was calculated by dividing number of convulsive seizures by the total number of seizures (convulsive proportion).

The mean seizure duration was calculated for five recording blocks, each being four days of duration. Days included in each block were as follows: block one: days three to six; block two: days seven to ten; block three: days 11 to 14; block four: days 15 to 18; and block five: days 19 to 21 . The mean seizure duration for each block was calculated by (a) first averaging the duration of all seizures that a rat had in a single recording session, (b) then averaging all that rat's session means over the block, and (c) determining the mean \pm the standard error of the mean (SEM) of the block averages from all rats. This was done to eliminate any bias from rats that had more frequent seizures or that were monitored more frequently.

The mean seizure frequency was also calculated for each of the recording blocks. This was calculated by (a) first determining the seizure frequency of each recording session, (b) averaging all the seizure frequencies across the block for each rat, and (c) determining the mean \pm SEM of the seizure frequency averages from all rats.

Seizure severity was also determined for each recording block, using the observations recorded during ECoG monitoring. Seizure severity was calculated by (a) first dividing number of convulsive seizures by the total number of seizures to give the proportion of seizures that were convulsive (convulsive proportion) for each recording session, (b) averaging all the convulsive proportions across the block for each rat, and (c) determining the mean \pm SEM of the convulsive proportions from all rats.

The difference in the median seizure duration and frequency between implanted and unimplanted control rats was determined for each block using a Wilcoxon-Mann-Whitney two-sample rank-sum test, performed using SigmaStat (v11.0, Systat Software, San Jose, CL, USA). 


\section{Results}

\subsection{In vitro drug release}

The total quantity of LEV released from the disk in each sample was determined using HPLC, and the mean and standard deviation calculated for each time point (Fig. 1). These results demonstrate that majority of drug release from the films is complete by day three, afterwhich point the drug release appeared to reach a plateau. Due to the large quantity of drug encapsulated, sensitive measurement techniques, small variations in disk size and potential evaporation of aCSF from the vials over time, there was a high degree in variation in the results.

\subsection{Electrocorticography}

Injection of tetanus toxin into the hippocampus resulted in a syndrome of spontaneous seizures resembling those seen in human temporal lobe epilepsy ${ }^{[8,9]}$. Rats began to exhibit spontaneous seizures 2-13 days following tetanus toxin injection. Seizures consisted of either non-convulsive episodes of behavioral arrest, staring and chewing, or convulsive events that progressed through behavioral arrest, facial clonus, bilateral forelimb clonus, rearing and falling. These clinical events correlated with epileptiform discharges on the ECoG that consisted of an initial phase of high frequency polyspike activity of increasing amplitude followed by a period of lower amplitude rhythmic spike and wave activity (see Fig. 2). The duration of each seizure was measured using the ECOG and was defined as the time (in seconds) from the beginning of polyspike activity to the termination of spike and wave activity prior to the post-ictal depression.

\subsection{Seizure duration}

Rats implanted with LEV-loaded sheets had significantly shorter seizures compared to control rats during block one $(p=0.024)$. Control rats had long seizures at the onset of the seizure syndrome and progressed to shorter seizures from day 7 onwards, whereas rats 
implanted with LEV sheets did not manifest longer seizures at the start of the seizure syndrome (Figure 3A).

\subsection{Seizure frequency}

Implanted rats did not have significantly fewer seizures than control rats at any time point. There was a trend towards fewer seizures in LEV-implanted rats during block one, however this difference was not significant $(p=0.078)$ (Figure 3B).

\subsection{Seizure severity}

All rats exhibited mostly non-convulsive seizures during the first days after tetanus toxin injection. One week after tetanus toxin injection, control rats rapidly progressed to exhibiting almost exclusively convulsive seizures whilst, in contrast, rats implanted with LEV-loaded sheets continued to have predominantly non-convulsive events, however this difference did not reach significance (proportion of seizures that were convulsive: control $=0.966 \pm 0.0218$; implanted $=0.470 \pm 0.145 ; p=0.086$, Fig. $3 C$ ). By the third monitoring block, seizures exhibited by control rats had settled and about half of the events were non-convulsive, similar to those exhibited by implanted rats during this block. This pattern continued until the end of monitoring at three weeks post-injection.

\section{Discussion}

Biodegradable polymer sheets containing a large amount of LEV were implanted above the motor and somatosensory cortices of epileptic rats with a hippocampal seizure focus. Implantation of the sheets led to shorter seizures and a trend towards fewer and less severe seizures in the first week after implantation compared to rats that did not receive the implant.

An important limitation of the current study is that a single group of control animals were investigated. These animals received an injection of tetanus toxin and a sham craniotomy, without the implantation of a polymer sheet. It is possible that the presence of the polymer 
sheet, irrespective of drug release, may have altered the function of the underlying brain or otherwise affected the intracranial environment such that seizures were ameliorated. An additional control group implanted with blank polymers, created in the same manner but with the drug load excluded or replaced with an inert sham substance, would assist in answering this question.

There are several possible explanations for the limited efficacy of the implants in this study. Firstly, the implants used in this investigation had a very high theoretical LEV load of $67 \%$ w/w.Our in vitro study demonstrated that such high drug loadings limited the ability of the polymer to regulate the release of LEV into the surrounding environment, producing large quantities of drug release for a short time period after implantation. This may explain why the implants produced a brief effect immediately after implantation.

Secondly, the sheets were implanted above the motor/somatosensory cortex whilst the seizure focus was within the hippocampus. In rats this distance is $4-5 \mathrm{~mm}$. Previous investigations have shown that substances released from intraparenchymally implanted polymers are able to penetrate around $3 \mathrm{~mm}^{[10-14]}$, suggesting that LEV released from the implants in our study would not have reached the seizure focus in the hippocampus in high concentrations. High concentrations of LEV in the motor and somatosensory cortices may have been able to slow or interrupt seizure propagation, leading to the observed reduction in seizure duration; however, the inadequate concentration in the hippocampus would have had no effect on seizure initiation and therefore on seizure frequency. 
This study demonstrates the ability of polymer-based brain implants to reduce the impact of seizures in the tetanus toxin model of mesial TLE. Whilst the results are modest, their limitations can be explained by the construction and location of the polymer. Indeed, the evidence of a trend towards a reduction in seizure frequency is promising given the distance of the implant from the seizure focus. The results indicate that further investigations into the use of biodegradable polymer-based implants for epilepsy are warranted. The incorporation of more potent anticonvulsants will permit the production of an implant with longer-term drug release without sacrificing efficacy, and should extend the success seen in the period immediately post-implantation. Additionally, implantation of the polymer at the seizure focus may reduce seizure frequency, including injectable polymer formulationssuch as microspheres. 


\section{Acknowledgements}

The authors gratefully acknowledge the donation of Levetiracetam from UCB Pharma, and the sponsorship of the Victorian Government through its Science Technology and Innovation Initiative administered by the Department of Industry, Innovation and Regional Development. The Bionic Ear Institute acknowledges the support it receives from the Victorian Government through its Operational Infrastructure Support Program and the Helen Macpherson Smith Trust. The authors also gratefully acknowledge Dean Freestone and Alan Lai for their assistance in programming the software for review of the EEG data. 


\section{References}

1. Halliday AJ, Cook MJ. Polymer-based drug delivery devices for neurological disorders. CNS Neurol Disord Drug Targets 2009; 8 (3):205-21.

2. McRae A, Hjorth S, Mason DW, Dillon L, Tice TR. Microencapsulated dopamine (DA)-induced restitution of function in 6-OHDA-denervated rat striatum in vivo: comparison between two microsphere excipients.J Neural Transplant Plast 1991; 2 (3-4):165-73.

3. McRae A, Dahlstrom A. Transmitter-loaded polymeric microspheres induce regrowth of dopaminergic nerve terminals in striata of rats with 6-OH-DA induced parkinsonism. Neurochem Int 1994; 25 (1):27-33.

4. Gouhier C, Chalon S, Venier-Julienne MC, Bodard S, Benoit J, Besnard J, Guilloteau D. Neuroprotection of nerve growth factor-loaded microspheres on the D2 dopaminergic receptor positive-striatal neurones in quinolinic acidlesioned rats: a quantitative autoradiographic assessment with iodobenzamide. Neurosci Lett 2000; 288 (1):71-5.

5. Menei P, Pean JM, Nerriere-Daguin V, Jollivet C, Brachet P, Benoit JP. Intracerebral implantation of NGF-releasing biodegradable microspheres protects striatum against excitotoxic damage.Exp Neurol 2000; 161 (1):25972.

6. Pean JM, Menei P, Morel O, Montero-Menei CN, Benoit JP. Intraseptal implantation of NGF-releasing microspheres promote the survival of axotomized cholinergic neurons.Biomaterials 2000; 21 (20):2097-101.

7. Arica B, Kas HS, Moghdam A, Akalan N, Hincal AA. Carbidopa/levodopaloaded biodegradable microspheres: in vivo evaluation on experimental Parkinsonism in rats.J Control Release 2005; 102 (3):689-97.

8. Doheny HC, Whittington MA, Jefferys JG, Patsalos PN. A comparison of the efficacy of carbamazepine and the novel anti-epileptic drug levetiracetam in the tetanus toxin model of focal complex partial epilepsy. $\mathrm{Br} \mathrm{J}$ Pharmacol 2002; 135 (6):1425-34. 
9. Finnerty GT, Jefferys JG. 9-16 Hz oscillation precedes secondary generalization of seizures in the rat tetanus toxin model of epilepsy.J Neurophysiol 2000; 83 (4):2217-26.

10. Krewson CE, Dause R, Mak M, Saltzman WM. Stabilization of nerve growth factor in controlled release polymers and in tissue.J Biomater Sci Polym Ed 1996; 8 (2):103-17.

11. Krewson CE, Klarman ML, Saltzman WM. Distribution of nerve growth factor following direct delivery to brain interstitium.Brain Res 1995; 680 (1-2):196206.

12. Saltzman WM, Mak MW, Mahoney MJ, Duenas ET, Cleland JL. Intracranial delivery of recombinant nerve growth factor: release kinetics and protein distribution for three delivery systems. Pharm Res 1999; 16 (2):232-40.

13. Bensadoun JC, Pereira de Almeida L, Fine EG, Tseng JL, Deglon N, Aebischer P. Comparative study of GDNF delivery systems for the CNS: polymer rods, encapsulated cells, and lentiviral vectors.J Control Release 2003; 87 (1-3):107-15.

14. Smith AL, Parsons CH, Lanyon RG, Bizley JK, Akerman CJ, Baker GE, Dempster AC, Thompson ID, King AJ. An investigation of the role of auditory cortex in sound localization using muscimol-releasing Elvax.Eur $\mathrm{J} N e u r o s c i$ 2004; 19 (11):3059-72. 


\section{Captions}

Figure 1. In vitro LEV release from biodegradable PLGA films.

Figure 2. Typical electrocorticograms recorded from tetanus-toxin injected rats during spontaneous seizures.

Figure 3.Efficacy of LEV-loaded polymer sheets in tetanus toxin rats. The figures illustrate the mean $( \pm$ SEM) of the seizure duration, frequency and severity in tetanus toxin injected rats with and without LEV-loaded implants. 